Palavras chave: Ergonomia Vibração mecânica Feller-Buncher Skidder Garra traçadora

Histórico: Recebido 20/01/2012 Aceito 14/04/2014

Keywords: Ergonomics Mechanical vibration Feller Buncher Skidder Slash grapple

Correspondência: roberto@feagri.unicamp.br
Simone Formigoni Almeida', Roberto Funes Abrahão', Mauro José Andrade Tereso'

\section{AVALIAÇÃO DA EXPOSIÇÃO OCUPACIONAL À VIBRAÇÃO DE CORPO INTEIRO EM MÁQUINAS DE COLHEITA FLORESTAL}

RESUMO: No Brasil, ainda são poucos os estudos que quantificam os níveis de exposição à vibração mecânica de corpo inteiro com máquinas florestais. Este estudo foi realizado na região de Campinas, em áreas de colheita de eucalipto de uma empresa de celulose e papel, objetivando quantificar os níveis de vibração aleatória a que estão expostos os operadores de três tipos de máquina de colheita de madeira - Feller-Buncher, Skidder e Garra Traçadora - durante a jornada de trabalho. Foi utilizado um medidor integrador triaxial Larson Davis modelo HVM 100 e os resultados analisados de acordo com as recomendações das normas ISO 263I-I: 1997 (no critério saúde) e Diretiva 2002/44 da Comunidade Européia. O Feller-Buncher apresentou uma magnitude de vibração equivalente de $1,12 \mathrm{~m} \cdot \mathrm{s}^{-2}$ (soma ponderada dos valores encontrados nos três eixos) sendo que, segundo a metodologia de Griffin (1998), o tempo máximo de exposição recomendado deveria ser de $4 \mathrm{~h} 47 \mathrm{~min}$. O Skidder apresentou uma magnitude de vibração equivalente de $1,95 \mathrm{~m} \cdot \mathrm{s}^{-2}$, onde, de acordo com a mesma metodologia, o tempo de exposição máxima não deveria ultrapassar 1 h35min. A Garra Traçadora apresentou os níveis mais próximos e dentro das recomendações das referidas normas: $0,99 \mathrm{~m} \cdot \mathrm{s}^{-2}$. Apesar de toda tecnologia empregada pelos fabricantes de máquinas de colheita florestal, os níveis de exposição às vibrações ainda estão além dos parâmetros recomendados pelas normas pertinentes.

\section{EVALUATION OF OCCUPATIONAL EXPOSURE TO WHOLE BODY VIBRATION IN FOREST HARVESTING MACHINES}

ABSTRACT: In Brazil there are few studies that quantify the levels of exposure to whole body mechanical vibration on forest harvesting machines. This study was conducted in the region of Campinas, in areas of eucalyptus harvest from a cellulose and paper company, to quantify the levels of random vibration to which operators are exposed to in three types of timber harvesting machines - Feller Buncher, Slash Grapple and Skidder - during the workday. A Larson Davis triaxial meter integrator model HVM 100 was used and the results compared to the recommendations of ISO 263 I-I: 1997 (in health criterion) and Directive 2002/44 of the European Community. The Feller-Buncher had a magnitude of vibration equivalent of $1.12 \mathrm{~m} \cdot \mathrm{s}^{-2}$, and the recommended maximum exposure time according Griffin's (1998) methodology should be 4h47min. The Skidder showed a magnitude of vibration equivalent of $1.95 \mathrm{~m} \cdot \mathrm{s}^{-2}$, where the maximum exposure should not exceed Ih35min. The Slash Grapple showed the levels near and within the recommendations of these standards: $0.99 \mathrm{~m} \cdot \mathrm{s}^{-2}$. Despite all the technology used by manufacturers of machinery for harvesting, levels of exposure to vibration are still beyond the parameters recommended by relevant standards. 


\section{INTRODUÇÃO}

O Brasil possui uma das maiores reservas florestais do mundo e tem desenvolvido as melhores tecnologias agrícolas para o cultivo de florestas em clima tropical, com extraordinários ganhos de produtividade.

Com o aumento da área reflorestada e da mecanização na colheita de madeira, iniciados há cerca de 20 anos, o setor florestal apresentou um crescimento significativo tanto para o mercado interno quanto para as exportações, contribuindo com a geração de mais de 3,9 milhões de empregos diretos e indiretos em toda cadeia produtiva da indústria de base florestal (ASSOCIAÇÃO BRASILEIRA DOS PRODUTORES DE FLORESTAS PLANTADAS - ABRAF, 2009). Dessa forma, tem ocorrido um crescente número de operadores de máquinas de colheita florestal expostos à vibração.

Esses indicativos acabam justificando os altos investimentos que o setor tem recebido, principalmente em recursos tecnológicos. Com a abertura do mercado de importações e incentivos do governo federal, as indústrias do setor florestal - com destaque para o segmento de celulose e papel - aumentaram seu contingente de máquinas próprias, principalmente para a colheita de pinus e eucalipto.

Disso decorre a importância dos estudos que vêm sendo realizados sobre os aspectos ergonômicos das máquinas de colheita florestal, a fim de melhorar as condições de trabalho dos operadores nacionais.

Fontana (2005), Lima et al. (2005), Minette et al. (2007) e Silva et al. (2003), entre outros, realizaram avaliações em diferentes tipos de máquinas de colheita florestal sobre diversos aspectos ergonômicos das máquinas, abordando, principalmente, questões de antropometria, visibilidade da área de trabalho e exposição dos operadores a agentes físicos como ruído, temperatura e vibração - este último avaliado subjetivamente, colhendo as impressões dos operadores.

Yamashita (2002) realizou avaliação quantitativa da exposição à vibração dos operadores de um harvester, de um slingshot, um feller-buncher e de um forwarder em situações reais de trabalho, visando a discutir os efeitos potenciais da exposição à vibração de corpo inteiro e às condições de segurança no local de trabalho. A autora concluiu que, com exceção do valor encontrado no eixo $x$ do feller-buncher e eixo $y$ do forwarder, os demais dados encontrados se apresentaram muito superiores aos estabelecidos na norma ISO 263I-I: 1985, usada como referência.

Cation et al. (2008) realizaram a avaliação dos níveis de vibração em sete skidders, em Ontário,
Canadá, adotando seis graus de liberdade (número de coordenadas independentes requerido para a descrição do seu movimento (THOMSON, 1978)), avaliando as vibrações lineares nos três eixos $X, Y$ e $Z$ e roll - rotação sobre o eixo $X$, pitch - rotação sobre o eixo $Y$ e yaw - rotação sobre o eixo $Z$. As medições foram realizadas com os acelerômetros instalados na interface operadorassento e nos chassis das máquinas, simultaneamente. Os resultados demonstraram que 0 assento dos skidders amplificaram as vibrações recebidas na interface assento-operador, quando comparadas aos valores correspondentes medidos nos chassis das máquinas, repetindo valores muito próximos de estudos realizados há vinte anos.

Os trabalhadores, sejam de qual setor forem, estão sujeitos a constrangimentos diversos, durante 0 desenrolar de suas atividades, que podem conduzi-los, principalmente: a erros de operação - comprometendo seu desempenho, sua segurança e dos demais e a problemas de saúde - física e emocional. A vibração mecânica é um tipo de constrangimento que atinge inúmeros trabalhadores.

A Convenção $n^{\circ} 148$ da Organização Internacional do Trabalho, em seu artigo 3, estabelece que “o termo 'vibrações' compreende toda vibração transmitida ao organismo humano por estruturas sólidas e que seja nociva à saúde ou contenha qualquer outro tipo de perigo" (BRASIL, 2002).

Grandjean (1998) comenta que as vibrações têm numerosos efeitos fisiológicos que abrangem, em pequena intensidade, músculos, circulação e respiração e, em grande intensidade, a percepção visual e produção psicomotora. lida (2003) afirma que as vibrações são particularmente danosas ao organismo nas frequências mais baixas, de $\mathrm{IHz}$ a $80 \mathrm{~Hz}$, podendo provocar lesões nos ossos, juntas e tendões.

Os limites e recomendações preventivas a respeito da exposição às vibrações estão previstos em normas internacionais que servem de referência para a legislação nacional pertinente ao assunto. As principais e mais referenciadas são as normas ISO 263 I - I: 1997 para vibrações de corpo inteiro; ISO 5349- I : 200 I para vibração localizada (mãos) e a Diretiva 2002/44 da Comunidade Europeia (DIRETIVA..., 2002; INTERNATIONAL ORGANIZATION for STANDARDZATION - ISO, 1997, 200I).

As normas ISO 263 I- I: I 997 e ISO 5349- I:200 I (ISO, 1997, 200I) definem a vibração de corpo inteiro em três variáveis: frequência $(\mathrm{Hz})$, aceleração máxima sofrida pelo corpo $\left(\mathrm{m} \cdot \mathrm{s}^{-2}\right)$ e direção do movimento, que é dada em três eixos espaciais: x (ântero-posterior) y (lateral; direita-esquerda) e z (longitudinal; pés-cabeça). 
A exposição às vibrações mecânicas é um dos mais importantes fatores de risco a que os operadores de máquinas de colheita florestal estão submetidos. Em função disso, este trabalho buscou quantificar os níveis de exposição dos operadores às vibrações mecânicas de corpo inteiro e comparar os resultados com as recomendações da norma ISO 263I-I: 1997 (no critério saúde) e da Diretiva 2002/44 da Comunidade Europeia (DIRETIVA..., 2002; ISO, 1997).

\section{MATERIAL E MÉTODOS}

Este estudo foi realizado em dois hortos de colheita de eucalipto, de setembro a novembro de 2010 , em uma empresa de celulose e papel, na região de Campinas - SP. O solo predominante da região é do tipo argiloso; o sistema de colheita da empresa é do tipo toras longas ou fuste.

Participaram, dessa pesquisa, 48 operadores de máquina de colheita florestal $(80 \%$ dos operadores da empresa). Esses operadores responderam a um questionário que procurou caracterizá-los com relação à idade, escolaridade, tempo na empresa e na função, buscando, também, avaliar sua percepção subjetiva acerca da exposição às vibrações mecânicas. Os operadores opinaram entre "bom, regular e ruim" sobre o sistema de amortecimento das máquinas e entre "rara, esporádica ou constante" sobre a sensação de desconforto relacionado à vibração.

A avaliação quantitativa envolveu 6 operadores (2 para cada modelo de máquina), operando suas máquinas, durante as medições, deslocando-se pelo talhão - de topografia irregular e sobre tocos. Os modelos avaliados foram: Feller-Buncher $860 \mathrm{C}$ Tigercat, ano $2006 \mathrm{com}$ rodado de esteiras, 17800 horas de uso, assento fixo, de espuma com amortecedor pneumático e regulagem da calibragem, velocidade de deslocamento no talhão de até $4,5 \mathrm{~km} \cdot \mathrm{h}^{-1}$; Skidder $635 \mathrm{D}$ Tigercat, ano 2009, com rodados de pneus $6 \times 6 \mathrm{com}$ calibragem dianteira em 30 libras e traseira de 35 libras, 4837 horas de uso, banco giratório em $100^{\circ} \mathrm{em}$ torno de seu eixo e amortecedor pneumático com regulagem da calibragem, velocidade durante o deslocamento no talhão de até $14 \mathrm{~km} \cdot \mathrm{h}^{-1}$; e Garra-Traçadora 320C L Caterpillar, ano 2007, com rodado de esteira, 14700 horas de uso, assento fixo, de espuma com amortecedor pneumático e regulagem de calibragem, velocidade ao deslocar-se pelo talhão de $3 \mathrm{~km} \cdot \mathrm{h}^{-1}$.

Para a avaliação da exposição de corpo inteiro, adotou-se norma ISO 263 I-I:1997 (ISO, 1997), que fixa um sistema de coordenadas com origem no ponto onde a vibração se incorpora ao corpo humano, ou seja, na interface entre a fonte vibratória e o corpo. $O$ método básico utilizado nas medições é o da aceleração ponderada, expressa em $\mathrm{m} \cdot \mathrm{s}^{-2}$, cujo valor total nas coordenadas ortogonais foi calculado pela equação 1 , sendo awx, awy e awz são as acelerações ponderadas dos eixos ortogonais, $x, y$ e $z$, respectivamente e $k x$, $k y$ e $k z$ são fatores multiplicadores, onde kx e ky = I,4 e kz = I,0 (ISO, 1997).

$$
a_{v}=\sqrt{k_{x}^{2} \cdot a_{w x}^{2}+k_{y}^{2} \cdot a_{w y}^{2}+k_{z}^{2} \cdot a_{w z}^{2}}
$$

Para determinar a relação entre os níveis de aceleração ponderada e a duração da exposição, com destaque para as exposições na faixa de 4 a 8 horas, para pessoas sentadas, utilizou-se o gráfico do anexo $B$ daquela norma (Figura 1), sendo que os resultados foram inseridos nas equações propostas por Griffin (1998) para determinação do limite de tempo superior de exposição (equações 2 e 3 , sendo $\mathrm{Tu}=$ o limite de tempo superior de exposição; $\mathrm{TI}=0$ limite de tempo inferior de exposição; e aw $=$ a aceleração ponderada $\mathrm{em} \mathrm{m} \cdot \mathrm{s}^{-2}$ ).

$$
\begin{aligned}
& \mathrm{T}_{\mathrm{u}}(\min )=10 \cdot\left[\frac{6}{\mathrm{aw}}\right]^{2} \\
& \mathrm{TI}(\min )=10 \cdot\left[\frac{3}{\mathrm{aw}}\right]^{2}
\end{aligned}
$$

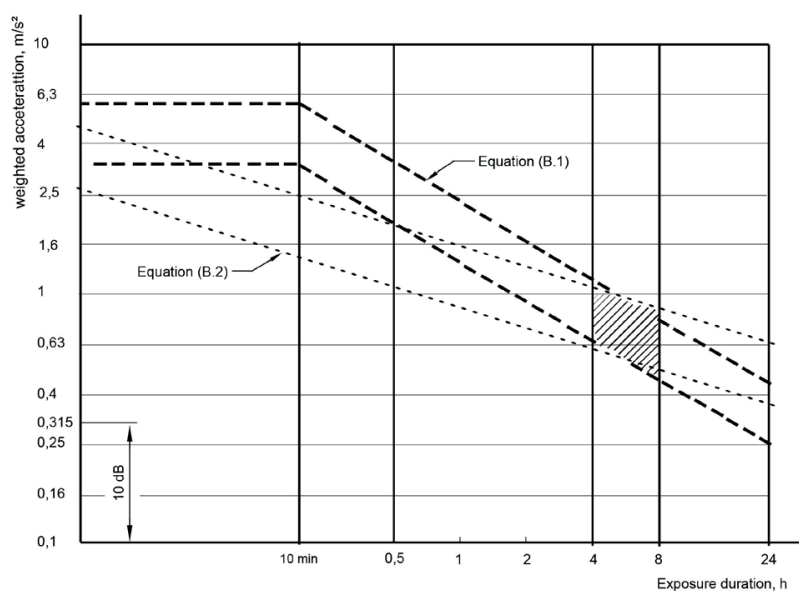

FIGURA 1 Gráfico guia com as zonas de precaução à saúde FIGURE 1 Health guidance caution zones. Fonte: ISO (1997) - Anexo B.

$\mathrm{Na}$ figura 1, a região em hachura indica "precauções em relação aos riscos potenciais à saúde"; a região acima indica "riscos prováveis à saúde" e a região abaixo indica que "os efeitos à saúde não têm sido claramente documentados e/ou observados objetivamente". 
A Diretiva 2002/44 da Comunidade Europeia também foi utilizada como referência para as avaliações da exposição às vibrações, indicando níveis de ação e limites de exposição, conforme a Tabela 1.

TABELA 1 Recomendações da Diretiva 2002/44 CE.

TABLE 1 Recommendation from Directive 2002/44/EC

\begin{tabular}{cc}
\hline Diretiva 2002/44/CE - limites de exposição e níveis de ação \\
\hline Parâmetro & Vibração de Corpo Inteiro \\
\hline \multirow{2}{*}{ Nível de Ação (NA) } & $0,5 \mathrm{~m} \cdot \mathrm{s}^{-2} \mathrm{~A}(8)^{(a)}$ ou \\
& $9,1 \mathrm{VDV}^{(\mathrm{b})}($ pior eixo) \\
\hline \multirow{2}{*}{ Limite de Exposição } & $1,15 \mathrm{~m} \cdot \mathrm{s}^{-2} \mathrm{~A}(8)^{(\mathrm{a})}$ ou \\
& $21 \mathrm{VDV}{ }^{(\mathrm{b})}$ (pior eixo) \\
\hline
\end{tabular}

(a) A avaliação da exposição à vibração de corpo inteiro (VCl) baseia-se na determinação da exposição diária $A(8)$ expressa pela aceleração equivalente para um período normalizado de 8 horas, obtida a partir da maior parcela dos valores eficazes, ou a parcela mais elevada do valor de dose da vibração (VDV), das acelerações ponderadas em frequência determinada segundo os três eixos ortogonais $\left(1,4 \mathrm{a}_{\mathrm{wx}}, 1,4\right.$ $a_{w y} 1 a_{w z}$ para trabalhadores sentados ou em pé), conforme capítulos 5, 6 e 7 e anexos A e B da ISO 2631-1:1997.

(b) VDV - Valor de Dose da Vibração, parâmetro a ser utilizado conforme ISO 2631- 1 quando há presença de picos ou choques significativos.

Fonte: Cunha (2004)

As medições dos níveis de vibração foram realizadas, utilizando-se um medidor integrador de vibração triaxial da marca Larson Davis, modelo HVM $100^{\circledR}$ que atende às normas ISO 804 I, ISO 263 I partes I e 2, ISO 5349 partes I e 2 e ISO I08 I9. Esse aparelho fornece a soma integrada de cada eixo e a somatória total da aceleração encontrada. $O$ acelerômetro foi fixado no assento das máquinas, por meio de um dispositivo almofadado preso com fitas adesivas e o aparelho HVM $100^{\circledR}$ foi colocado na cintura dos operadores.

As medições ocorreram no transcorrer das atividades normais do operador, em dois dias diferentes, com um intervalo de 20 dias entre eles, buscando registrar a exposição à vibração em situações reais de trabalho. Os dados foram registrados por cerca de 15 a 20 minutos para cada operador, de forma a cobrir os ciclos de operação de cada máquina, considerando-se a variável dependente aceleração equivalente (nos eixos $X, Y$ e Z ) e as variáveis independentes deslocamento descarregado e carregado na operação do Feller-Buncher e do Skidder e corte e empilhamento na operação da Garra Traçadora.

\section{RESULTADOS E DISCUSSÃO}

Com relação à população envolvida, todos os 48 respondentes são do sexo masculino; grande parte com idade predominante entre 30 e 39 anos (35\%) e 40 a 49 anos (40\%). Todos estão na empresa há mais de 15 anos. Como operadores de máquina de colheita, $44 \%$ ocupam essa função há mais de 15 anos. São operadores experientes e oferecem suporte aos ingressantes mais recentes. Pouco mais da metade dos operadores (52\%) têm baixa escolaridade, apresentando o ensino fundamental completo (6\%) ou incompleto (46\%); $40 \%$ apresentam ensino médio completo (incluindo nível técnico) e $8 \%$ incompleto.

Segundo o questionário respondido pelos operadores, a maioria está satisfeita com o sistema de amortecimento de suas máquinas. Porém, um número significativo dos operadores (42\%) considerou o Skidder regular e a sensação de desconforto foi constante para $25 \%$ destes (Tabela 2).

$\mathrm{Na}$ avaliação quantitativa dos resultados procurou-se separá-los por tipo de máquina e os ciclos que compõem cada tarefa. As medições cobriram as atividades normais de trabalho, sem interrupção ou distinção entre as atividades principais (ciclo propriamente dito) e atividades complementares da tarefa - pequenas interrupções para atender ao rádio ou pequenas manobras para ajuste das toras.

Considerou-se como ciclo completo de operação do Feller-Buncher o deslocamento descarregado pelo talhão até o local de corte, o corte de 6 a 7 árvores acumuladas no cabeçote e o deslocamento carregado para posterior tombamento. Os registros totalizaram II74 segundos de operação. $O$ deslocamento descarregado ocorreu a uma velocidade média de $4,5 \mathrm{~km} \cdot \mathrm{h}^{-1}$ e o deslocamento carregado a uma velocidade média de $2,0 \mathrm{~km} \cdot \mathrm{h}^{-1}$.

TABELA 2 Caracterização subjetiva das condições vibratórias. TABLE 2 Characterization of subjective vibratory conditions.

\begin{tabular}{|c|c|c|c|c|c|c|c|c|c|}
\hline \multirow{2}{*}{$\frac{\text { Vibração }}{\text { Item avaliado }}$} & \multicolumn{9}{|c|}{ Classificação (\%) } \\
\hline & \multicolumn{3}{|c|}{ Skidder $635 \mathrm{D}$} & \multicolumn{3}{|c|}{ Feller-Buncher $860 \mathrm{C}$} & \multicolumn{3}{|c|}{ Garra Traçadora 320C L } \\
\hline \multirow{2}{*}{ Amortecimento } & Bom & Regular & Ruim & Bom & Regular & Ruim & Bom & Regular & Ruim \\
\hline & 58 & 42 & - & 91 & 9 & - & 60 & 20 & 20 \\
\hline \multirow{2}{*}{ Sensação de desconforto } & Constante & Esporádica & Rara & Constante & Esporádica & Rara & Constante & Esporádica & Rara \\
\hline & 25 & 8 & 67 & - & 9 & 91 & 20 & 20 & 60 \\
\hline
\end{tabular}


Para o ciclo medido do Skidder, considerou-se o deslocamento descarregado, carregamento da garra com dois feixes deárvores ( 50 árvores em média), deslocamento carregado (arraste), manobras e depósito dos feixes na bordadura do talhão. Foram medidos II 56 segundos de exposição. $O$ deslocamento descarregado ocorreu a uma velocidade média de $14 \mathrm{~km} \cdot \mathrm{h}^{-1}$ e o deslocamento carregado a uma velocidade média de $6 \mathrm{~km} \cdot \mathrm{h}^{-1}$.

Com a Garra Traçadora, o ciclo considerado para as medições foi de traçamento composto de 7 cortes, incluindo pequenas manobras (sem deslocamento), abrir garra e pegar um feixe, puxar o feixe colocando-o na pilha de corte, soltá-lo sobre a pilha, segurá-lo na altura de corte e cortá-lo. Foram medidos cinco ciclos de 7 cortes, totalizando 910 segundos de medição.

As tabelas 3, 4 e 5 apresentam: os níveis de aceleração equivalente ponderada para os eixos $\mathrm{X}, \mathrm{Y}$ e Z; a somatória ponderada (SUM) dos três eixos para cada máquina; a magnitude de vibração equivalente total da exposição (SUM em $\mathrm{m} \cdot \mathrm{s}^{-2}$ ); e o VDV total (Valor de Dose da Vibração, parâmetro a ser considerado - conforme ISO (1997) - quando há presença de picos ou choques significativos, em $\left.\mathrm{m} \cdot \mathrm{s}^{-1,75}\right)$.

A avaliação do Feller-Buncher resultou em uma magnitude de vibração equivalente total de $\mathrm{I}, 12 \mathrm{~m} \cdot \mathrm{s}^{-2} \mathrm{e}$ 。 VDV total encontrado foi de $8,75 \mathrm{~m} \cdot \mathrm{s}^{-1,75}$. Contrariando a opinião dos operadores, em que $82 \%$ consideraram como "bom" o sistema de amortecedor de vibração, o Feller-Buncher $860 \mathrm{C}$ apresentou níveis vibratórios acima da zona de cautela do gráfico do anexo B da ISO 263II: 1997 (ISO, 1997), tendo o eixo $X$ apresentado a maior variação. O NA/VLE (nível de ação do Valor Limite de Exposição) também se mostrou acima do recomendado pela diretiva europeia. Com relação a Diretiva 2002/44 CE a magnitude de vibração equivalente está aceitável em relação ao VLE (valor limite de exposição); porém, o NAVVLE (Nível de ação do VLE) encontra-se acima das recomendações. Já, o VDV total encontra-se abaixo do VDV e do NA-VDV recomendados (Tabela 3).

A avaliação do Skidder resultou em uma magnitude de vibração equivalente (SUM total) de I,95 $\mathrm{m} \cdot \mathrm{s}^{-2} \mathrm{e}$ o VDV total encontrado foi de $7,01 \mathrm{~m} \cdot \mathrm{s}^{-1,75}$, acima da recomendação da ISO 263 I-I: I 997 . O Skidder 635 D apresentou os maiores níveis de aceleração ponderada, comparativamente às outras duas máquinas avaliadas, coincidindo com as impressões dos operadores desse modelo, sendo o eixo Y o que apresentou maior variação em $75 \%$ da tarefa. De acordo com a diretiva europeia, o VLE e - NA/VLE também estavam acima das recomendações; já, o VDV total encontrado mostrou-se abaixo do VDV e do NA-VDV recomendados por essa diretiva (Tabela 4).

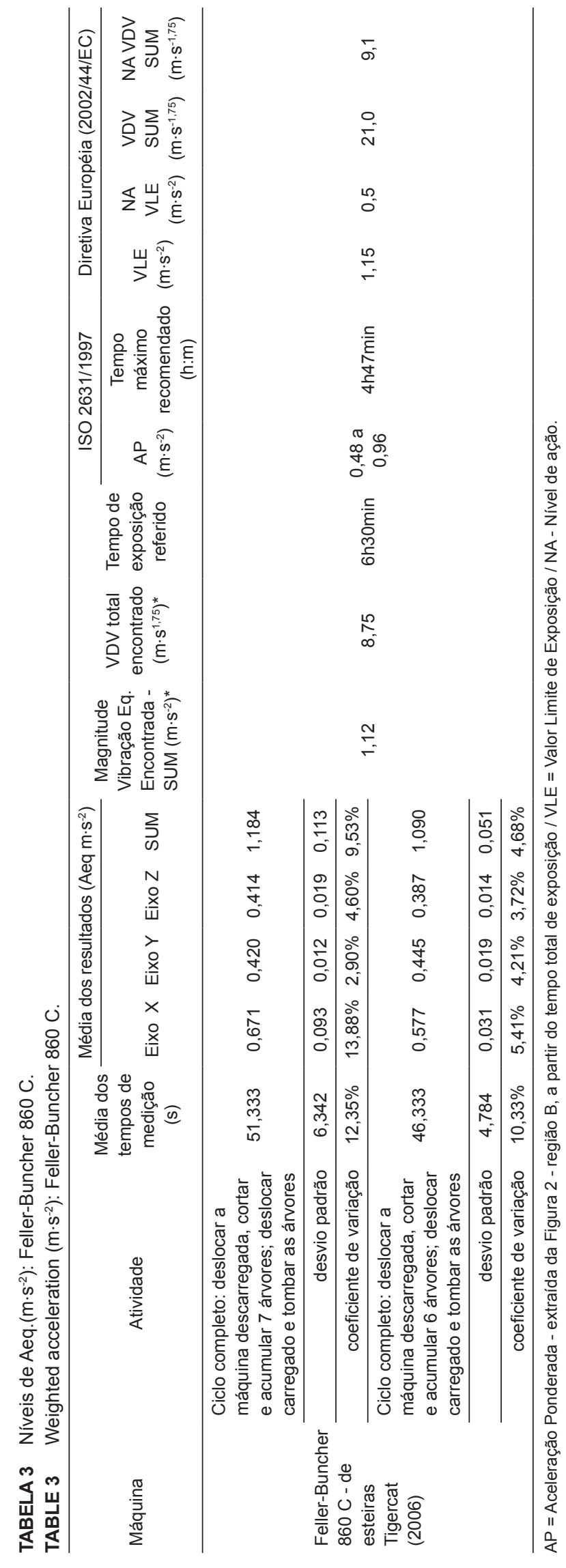




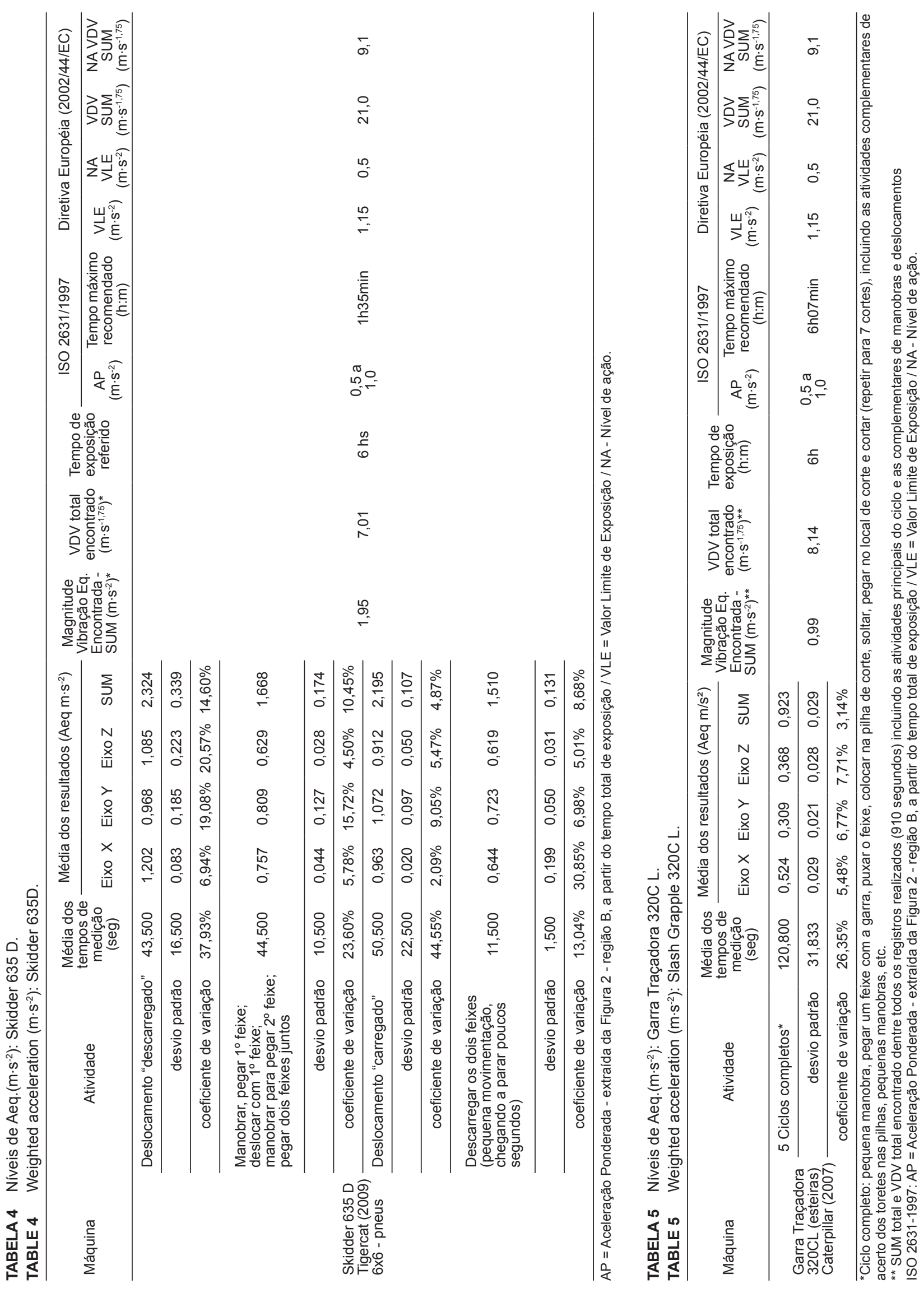


A avaliação da Garra Traçadora resultou em uma magnitude de vibração equivalente (SUM total) de $0,99 \mathrm{~m} \cdot \mathrm{s}^{-2}$ e o VDV total encontrado foi de 8,14 $\mathrm{m} \cdot \mathrm{s}^{-1,75}$, ficando entre o nível mínimo e máximo da recomendação da ISO 2631-I:1997. A Garra Traçadora 320C $L$ apresentou os níveis mais seguros com referência a ISO-263I-I, embora o nível de ação (NA) para o Valor Limite de Exposição (VLE) esteja acima do recomendado pela diretiva 2002/44/CE; já, o VDV total encontrado mostrou-se abaixo do VDV e do NA-VDV recomendados pela diretiva europeia (Tabela 5).

$\mathrm{Na}$ Figura 2, apresenta-se uma melhor visualização dos resultados encontrados com a projeção das medições dos níveis de vibração equivalente, realizadas nos três modelos de máquinas envolvidas neste estudo, e os tempos máximos encontrados com a equação de Griffin em relação ao Gráfico do Anexo B da norma ISO 263 I-I (ISO, I997).

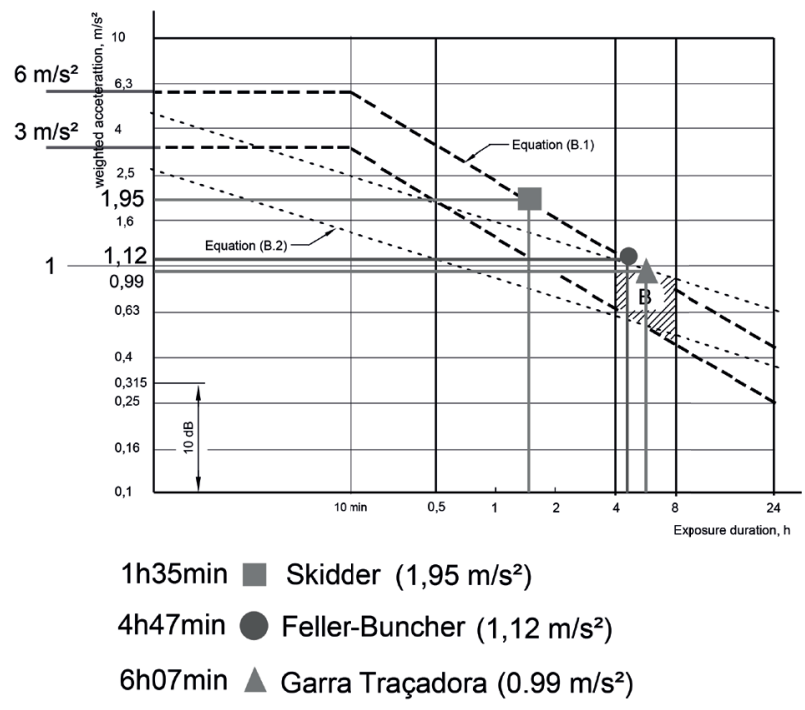

FIGURA 2 Resultados projetados no gráfico guia da ISO 26311:1997.

FIGURE 2 Projected results on the ISO 2631-1:1997 chart.

\section{CONCLUSÕES}

Pôde-se constatar que a atividade laboral do operador de máquina florestal é uma tarefa complexa, que demanda muito tempo em uma mesma postura (sentada) com o agravante da vibração recebida durante praticamente $75 \%$ da jornada diária. As tarefas apresentaram alta exigência cognitiva e motora, com movimentos de mãos e punhos simultâneos, porém assimétricos; curtos e leves, porém com alto índice de repetitividade.
A indústria de máquinas florestais vêm intensificando o desenvolvimento e aplicação de novas tecnologias, a fim de proporcionar maior conforto e segurança aos seus operadores, mas a organização do trabalho se mostra de fundamental importância na preservação da saúde do trabalhador. Maior número de pausas e exercícios que compensem os esforços da musculatura mais solicitada durante a jornada de trabalho têm sido amplamente estudados e seus benefícios comprovados. Nota-se, que apesar de toda tecnologia envolvida em cada máquina avaliada, os achados relativos à exposição ocupacional dos operadores às vibrações mecânicas se mostraram em patamares que suscitam ações mais eficazes, podendose considerar, inclusive, a diminuição da jornada de trabalho.

\section{AGRADECIMENTOS}

\author{
Agradecemos à FAPESP, pelo apoio e \\ financiamento deste estudo.
}

\section{REFERÊNCIAS}

ASSOCIAÇÃO BRASILEIRA DOS PRODUTORES DE FLORESTAS PLANTADAS. Anuário estatístico da ABRAF: ano base 2008. Brasília, 2009.

BRASIL. Convenções da OIT. Brasília: Ministério do Trabalho e Emprego, 2002.

CATION, S.; JACK, R.; OLIVER, M.; DICKEY, J. P.; LEE-SHEE, N. K. Six degree of freedom whole-body vibration during forestry skidder operations. International Journal of Industrial Ergonomics, Cambridge, v. 38, p. 739-757, Oct. 2008.

CUNHA, I. A. Exposição ocupacional às vibrações mecânicas: considerações sobre os principais critérios legais e técnicos. Revista ABHO, São Paulo, ano 3, n. 8, p. 9-12, mar. 2004.

DIRETIVA 2002/44/ CE do Parlamento Europeu e do Conselho. Jornal Oficial das Comunidades Européias, Lisboa, n. 177, p. 13-19, jun. 2002.

FONTANA, G. Avaliação ergonômica do projeto interno de cabines de forwarders e skidders. 2005. 80 p. Dissertação (Mestrado em Agronomia) - Escola Superior de Agricultura “Luiz de Queiroz”, Piracicaba, 2005.

GRANDJEAN, E. Manual de ergonomia: adaptando o trabalho ao homem. 4. ed. Porto Alegre: Bookman, 1998. $338 \mathrm{p}$. 
GRIFFIN, M. J. A comparison of standardized methods for predicting the hazards of whole-body vibration and repeated shocks. Journal of Sound and Vibration, Sheffield, v. 215, p. 883-914, 1998.

GUÉRIN, F;; LAVILLE, A.; DANIELLOU, F; DURAFFOURG, J.; KERGUELEN, A. Compreender o trabalho para transformá-lo: a prática da ergonomia. São Paulo: E. Blücher, 200I. 200 p.

IIDA, I. Ergonomia: projeto e produção. São Paulo: E. Blücher, 2003. $465 \mathrm{p}$.

INTERNATIONALORGANIZATIONFORSTANDARDZATION. ISO 263I-I: mechanical vibration and shock: evaluation exposure to whole-body vibration: part I, general requirements. Geneva, 1997. 32 p.

INTERNATIONAL ORGANIZATION FOR STANDARDZATION. ISO 5349-I: mechanical vibration: measurement and evaluation of human exposure to hand-transmitted vibration: part I, general requirements. Geneva, 200I. 15 p.
LIMA, J. S. S.; SOUZA, A. P.; MACHADO, C. C.; OLIVEIRA, R. B. Avaliação de alguns fatores ergonômicos nos tratores Feller-buncher e Skidder utilizados na colheita de madeira. Revista Árvore, Viçosa, v. 29, n. 2, p. 29I-298, 2005.

MINETTE, L. J.; SILVA, E. P.; SOUZA, A. P.; SILVA, K. R. Avaliação dos níveis de ruído, luz e calor em máquinas de colheita florestal. Revista Brasileira de Engenharia Agrícola e Ambiental, Campina Grande, v. II, n. 6, p. 664-667, 2007.

SILVA, C. B. da; SANT'ANNA, C. M.; MINETTI, L. J. Avaliação ergonômica do feller-buncher utilizado na colheita de eucalipto. Cerne, Lavras, v. 9, n. I, p. II9-127, 2003.

THOMSON, W. T. Teoria da vibração com aplicações. Rio de Janeiro: Interciência, 1978.

YAMASHITA, R. Y. Avaliação das condições de trabalho e da exposição à vibração do operador de máquinas na colheita florestal. 2002. 55 p. Dissertação (Mestrado em Ciências Florestais) - Escola Superior de Agronomia "Luiz de Queiroz”, Piracicaba, 2002. 